\title{
Complete moment convergence of extended negatively dependent random variables
}

\author{
Mingzhu Song ${ }^{1}$ and Quanxin Zhu ${ }^{2^{*}}$ (D)
}

"Correspondence: zqx22@126.com ${ }^{2}$ MOE-LCSM, School of Mathematics and Statistics, Hunan Normal University, Changsha, China Full list of author information is available at the end of the article

\begin{abstract}
In this paper, some results on the complete moment convergence of extended negatively dependent (END) random variables are established. The results in the paper improve and extend the corresponding ones of Qiu et al. (Acta Math. Appl. Sin. 40(3):436-448, 2017) under some weaker conditions. Our results also improve and extend the related known works in the literature.
\end{abstract}

MSC: $60 \mathrm{~F} 15$

Keywords: END random variables; Weighted sums; Complete convergence; Complete moment convergence

\section{Introduction}

In many statistical models, it is not reasonable to assume that random variables are independent, and so it is very meaningful to extend the concept of independence to dependence cases. One important dependence sequence of these dependences is extended negatively dependent (END) random variables, we recall the concept of END random variables as follows.

Definition 1.1 The random variables $\left\{X_{n}, n \geq 1\right\}$ are said to be extended negatively dependent (END) random variables if there exists a positive constant $M>0$ such that both

$$
P\left(X_{1}>x_{1}, X_{2}>x_{2}, \ldots, X_{n}>x_{n}\right) \leq M \prod_{i=1}^{n} P\left(X_{i}>x_{i}\right)
$$

and

$$
P\left(X_{1} \leq x_{1}, X_{2} \leq x_{2}, \ldots, X_{n} \leq x_{n}\right) \leq M \prod_{i=1}^{n} P\left(X_{i} \leq x_{i}\right)
$$

hold for each $n \geq 1$ and all real $x_{1}, x_{2}, \ldots, x_{n}$.

(c) The Author(s) 2020. This article is licensed under a Creative Commons Attribution 4.0 International License, which permits use sharing, adaptation, distribution and reproduction in any medium or format, as long as you give appropriate credit to the original author(s) and the source, provide a link to the Creative Commons licence, and indicate if changes were made. The images or other third party material in this article are included in the article's Creative Commons licence, unless indicated otherwise in a credit line to the material. If material is not included in the article's Creative Commons licence and your intended use is not permitted by statutory regulation or exceeds the permitted use, you will need to obtain permission directly from the copyright holder. To view a copy of this licence, visit http://creativecommons.org/licenses/by/4.0/. 
The concept of END random variables was introduced by Liu [2]. Obviously, END random variables $(M=1)$ imply NOD (negatively orthant dependent) random variables (JoagDev and Proschan [3]). Liu [2] pointed out that the END random variables are more comprehensive, and they can reflect not only negative dependence random variables but also positive ones, to some extent. Joag-Dev and Proschan [3] once pointed out that NOD random variables imply NA (negatively associated) random variables, but NA random variables do not imply NOD random variables, so END random variables imply NA random variables. Thus, it is interesting to investigate convergence properties for END random variables.

After the appearance of Liu [2], many scholars have focused on the properties of END random variables, and a lot of results have been gained. For example, Liu [4] studied necessary and sufficient conditions for moderate deviations of dependent random variables with heavy tails; Chen et al. [5] established strong law of large numbers for END random variables; Wu and Guan [6] presented convergence properties of the partial sums of END random variables; Shen [7] presented probability inequalities for END sequence and their applications; Wang and Wang [8] investigated large deviations for random sums of END random variables; Wang et al. and Qiu et al. [9-13] studied complete convergence of END random variables, etc.

The complete convergence plays a very important role in the probability theory and mathematical statistics. The concept of complete convergence was introduced by Hsu and Robbins [14] as follows: A sequence $\left\{U_{n}, n \geq 1\right\}$ of random variables is said to converge completely to a constant $\theta$ if, for $\forall \varepsilon>0, \sum_{n=1}^{\infty} P\left(\left|U_{n}-\theta\right|>\varepsilon\right)<\infty$. In view of the BorelCantelli lemma, the complete convergence implies that $U_{n} \rightarrow \theta$ almost surely. Therefore, complete convergence is a very important tool in establishing almost sure convergence for partial of random variables as well as weighted sums of random variables.

Let $\left\{X_{n}, n \geq 1\right\}$ be a sequence of random variables, $a_{n}>0, b_{n}>0, \gamma>0$. If for $\forall \varepsilon>0$, $\sum_{n=1}^{\infty} a_{n} E\left\{b_{n}^{-1}\left|X_{n}\right|-\varepsilon\right\}_{+}^{\gamma}<\infty$, then $\left\{X_{n}, n \geq 1\right\}$ is called the complete moment convergence (Chow [15]). It is well known that complete moment convergence implies complete convergence, i.e., the complete moment convergence is more general than complete convergence. The following result is from Chow [15].

Theorem A Let $r>1,1 \leq p<2,\left\{X, X_{n}, n \geq 1\right\}$ be a sequence of independent identically distributed random variables and $E X_{1}=0$, if $E\left\{\left|X_{1}\right|^{r p}+\left|X_{1}\right| \log \left(1+\left|X_{1}\right|\right)\right\}<\infty$, then

$$
\sum_{n=1}^{\infty} n^{r-2-1 / p} E\left\{\left|\sum_{i=1}^{n} X_{i}\right|-\varepsilon n^{1 / p}\right\}_{+}<\infty, \quad \forall \varepsilon>0 .
$$

It should be noted that Theorem A has been extended and improved by many scholars (see [16-19]).

Recently, Chen and Sung [20] obtained complete and complete moment convergence of $\rho$-mixing random variables, and Qiu et al. [1] obtained the following complete moment convergence for weighted sums of END random variables. 
Theorem B Let $r>1,1 \leq p<2, \lambda>0, \alpha>1, \beta>1$ with $1 / \alpha+1 / \beta=1 / p$. Let $\left\{a_{n i}, 1 \leq i \leq\right.$ $n, n \geq 1$ \} be an array of constants satisfying

$$
\sum_{i=1}^{n}\left|a_{n i}\right|^{a} \leq D n, \quad \forall n \geq 1
$$

where $D$ is a positive constant. $\left\{X, X_{n}, 1 \leq n\right\}$ is a sequence of identically distributed END random variables with $E X=0$. If

$$
\begin{cases}E|X|^{(r-1) \beta}<\infty & \text { if } \alpha<r p, \lambda<(r-1) \beta, \\ E|X|^{(r-1) \beta} \log (1+|X|)<\infty & \text { if } \alpha=r p, \lambda<(r-1) \beta, \\ E|X|^{(r-1) \beta} \log (1+|X|)<\infty & \text { if } \alpha<r p, \lambda=(r-1) \beta, \\ E|X|^{(r-1) \beta} \log ^{2}(1+|X|)<\infty & \text { if } \alpha=r p, \lambda=(r-1) \beta, \\ E|X|^{r p}<\infty & \text { if } \alpha>r p, \lambda<r p, \\ E|X|^{r p} \log (1+|X|)<\infty & \text { if } \alpha>r p, \lambda=r p, \\ E|X|^{\lambda}<\infty & \text { if } \lambda>\max \{r p,(r-1) \beta\}, \\ & \text { when } \alpha>r p, \text { assume } \lambda<\alpha,\end{cases}
$$

then

$$
\sum_{n=1}^{\infty} n^{r-2-\lambda / p} E\left\{\max _{1 \leq k \leq n}\left|\sum_{i=1}^{k} a_{n i} X_{i}\right|-\varepsilon n^{1 / p}\right\}_{+}^{\lambda}<\infty, \quad \forall \varepsilon>0 .
$$

In this article, our goal is to further study complete moment convergence for weighted sums of END random variables with suitable conditions. By using the truncated method, we obtain a novel result, which extends that in Qiu et al. [1] under some weaker conditions. Our result also improves and extends those in Chen and Sung [20], Sung [21], and Qiu and Xiao [22].

The layout of this paper is as follows. Main results and some lemmas are provided in Sect. 2. Proofs of the main results are given in Sect. 3. Throughout the paper, the symbol $C$ denotes a positive constant, which may take different values in different places. $I(A)$ is the indicator function of an event $A$.

\section{Main results and some lemmas}

Theoremm 2.1 Let $r>1,1 \leq p<2, \lambda>0, \alpha>0, \beta>0$ with $1 / \alpha+1 / \beta=1 / p$. Let $\left\{a_{n i}, 1 \leq i \leq\right.$ $n, n \geq 1\}$ be an array of constants satisfying (1.1). $\left\{X, X_{n}, n \geq 1\right\}$ is a sequence of identically distributed $E N D$ random variables with $E X=0$. Assume that one of the following conditions holds:

(1) If $\alpha<r p$, then

$$
\begin{cases}E|X|^{(r-1) \beta}<\infty & \text { if } \lambda<(r-1) \beta, \\ E|X|^{(r-1) \beta} \log (1+|X|)<\infty & \text { if } \lambda=(r-1) \beta, \\ E|X|^{\lambda}<\infty & \text { if } \lambda>(r-1) \beta .\end{cases}
$$


(2) If $\alpha=r p$, then

$$
\begin{cases}E|X|^{(r-1) \beta} \log (1+|X|)<\infty & \text { if } \lambda \leq(r-1) \beta=r p \\ E|X|^{\lambda}<\infty & \text { if } \lambda>(r-1) \beta=r p\end{cases}
$$

(3) If $\alpha>r p$, then

$$
\begin{cases}E|X|^{r p}<\infty & \text { if } \lambda<r p \\ E|X|^{r p} \log (1+|X|)<\infty & \text { if } \lambda=r p \\ E|X|^{\lambda}<\infty & \text { if } \lambda>r p\end{cases}
$$

Then

$$
\sum_{n=1}^{\infty} n^{r-2-\lambda / p} E\left\{\max _{1 \leq k \leq n}\left|\sum_{i=1}^{k} a_{n i} X_{i}\right|-\varepsilon n^{1 / p}\right\}_{+}^{\lambda}<\infty, \quad \forall \varepsilon>0
$$

Conversely, if (2.4) holds for any array $\left\{a_{n i}, 1 \leq i \leq n, n \geq 1\right\}$ satisfying (1.1), then $E X=0$, $E|X|^{(r-1) \beta}<\infty, E|X|^{r p}<\infty$.

Remark 2.1 The Rademacher-Menshov inequality is only used in the proof process of Theorem 2.1. The results in this paper still hold for random variable satisfying Rosenthal's inequality. Therefore, our results improve and extend the result of Chen and Sung [20].

Remark 2.2 In this paper, the conditions of Theorem 2.1 are weaker than those in Theorem 1.1 of Qiu et al. [1], and the condition of "if $\alpha>r p$, assume $\lambda<\alpha$ (Qiu et al. [1])" is not necessary for (2.4) in our paper. Therefore our results improve and extend the result of Qiu et al. [1]. It is worth pointing out that the method applied in this article is different from that in Qiu et al. [1].

To prove Theorem 2.1 of the paper, we need the following important lemmas.

Lemma 2.1 (Qiu [22]; Rademacher-Menshov inequality) Let $p>1,\left\{X_{n}, n \geq 1\right\}$ be a sequence of END random variables with $E X_{n}=0$ and $E\left|X_{n}\right|^{p}<\infty$. Then there exists a positive constant $C_{p}$ only depending on $p$ such that

$$
\begin{aligned}
& E\left(\max _{1 \leq j \leq n}\left|\sum_{i=1}^{j} X_{i}\right|^{p}\right) \leq C_{p} \log ^{p} n \sum_{i=1}^{n} E\left|X_{i}\right|^{p}, \quad 1<p \leq 2, \\
& E\left(\max _{1 \leq j \leq n}\left|\sum_{i=1}^{j} X_{i}\right|^{p}\right) \leq C_{p} \log ^{p} n\left\{\sum_{i=1}^{n} E\left|X_{i}\right|^{p}+\left(\sum_{i=1}^{n} E\left|X_{i}\right|^{2}\right)^{p / 2}\right\}, \quad p>2 .
\end{aligned}
$$

Lemma 2.2 (Qiu [22]) Let $p \geq 1,\left\{X_{n}, n \geq 1\right\}$ be a sequence of END random variables with $E X_{n}=0$ and $E\left|X_{n}\right|^{p}<\infty$. Then there exists a positive constant $C_{p}$ only depending on $p$ such 
that

$$
\begin{aligned}
& E\left(\left|\sum_{i=1}^{n} X_{i}\right|^{p}\right) \leq C_{p} \sum_{i=1}^{n} E\left|X_{i}\right|^{p}, \quad 1 \leq p<2, \\
& E\left(\left|\sum_{i=1}^{n} X_{i}\right|^{p}\right) \leq C_{p}\left\{\sum_{i=1}^{n} E\left|X_{i}\right|^{p}+\left(\sum_{i=1}^{n} E\left(X_{i}\right)^{2}\right)^{p / 2}\right\}, \quad p \geq 2 .
\end{aligned}
$$

Lemma 2.3 (Liu [2]) Let $\left\{X_{n}, n \geq 1\right\}$ be a sequence of END random variables. If $f_{1}, f_{2}, \ldots, f_{n}$ are all nondecreasing (or nonincreasing) functions, then random variables $f_{1}\left(X_{1}\right), f_{2}\left(X_{2}\right), \ldots$, $f_{n}\left(X_{n}\right)$ are still END random variables.

Lemma 2.4 (Wu [23]) Let $\left\{X_{n}, n \geq 1\right\}$ and $\left\{Y_{n}, n \geq 1\right\}$ be sequences of random variables, for any $q>r>0, \varepsilon>0, a>0$, then

$$
\begin{aligned}
E\left(\max _{1 \leq k \leq n}\left|\sum_{i=1}^{k}\left(X_{i}+Y_{i}\right)\right|-\varepsilon a\right)_{+}^{r} \leq & C_{r}\left(\frac{1}{\varepsilon^{q}}+\frac{r}{q-r}\right) \frac{1}{a^{q-r}} E\left(\max _{1 \leq k \leq n}\left|\sum_{i=1}^{k} X_{i}\right|^{q}\right) \\
& +C_{r} E\left(\max _{1 \leq k \leq n}\left|\sum_{i=1}^{k} Y_{i}\right|^{r}\right),
\end{aligned}
$$

where $C_{r}=1$ if $0<r \leq 1$ or $C_{r}=2^{r-1}$ if $r>1$.

Chen and Sung [20] obtained the following theorems (see Lemmas 2.5-2.7).

Lemma 2.5 (Chen [20]) Let $r>1,1 \leq p<2, \alpha>0, \beta>0$ with $1 / \alpha+1 / \beta=1 / p$. Let $\left\{a_{n i}, 1 \leq\right.$ $i \leq n, n \geq 1\}$ be an array of constants satisfying (1.1). $X$ is a random variable, then

$$
\sum_{n=1}^{\infty} n^{r-2} \sum_{i=1}^{n} P\left(\left|a_{n i} X\right|>n^{1 / p}\right) \leq \begin{cases}C E|X|^{(r-1) \beta} & \text { if } \alpha<r p \\ C E|X|^{(r-1) \beta} \log (1+|X|) & \text { if } \alpha=r p \\ C E|X|^{r p} & \text { if } \alpha>r p\end{cases}
$$

Lemma 2.6 (Chen [20]) Let $r>1,1 \leq p<2, \alpha>0, \beta>0$ with $1 / \alpha+1 / \beta=1 / p$. Let $\left\{a_{n i}, 1 \leq\right.$ $i \leq n, n \geq 1\}$ be an array of constants satisfying (1.1). If $X$ is a random variable, then for any $\nu>\max \{\alpha,(r-1) \beta\}$

$$
\sum_{n=1}^{\infty} n^{r-2-v / p} \sum_{i=1}^{n} E\left|a_{n i} X\right|^{v} I\left(\left|a_{n i} X\right| \leq n^{1 / p}\right) \leq \begin{cases}C E|X|^{(r-1) \beta} & \text { if } \alpha<r p \\ C E|X|^{(r-1) \beta} \log (1+|X|) & \text { if } \alpha=r p \\ C E|X|^{r p} & \text { if } \alpha>r p\end{cases}
$$

Lemma 2.7 (Chen [20]) Let $\lambda>0, r>1,1 \leq p<2, \alpha>0, \beta>0$ with $1 / \alpha+1 / \beta=1 / p$. Let $\left\{a_{n i}, 1 \leq i \leq n, n \geq 1\right\}$ be an array of constants satisfying (1.1) and $X$ be a random variable. Then the following statements hold: 
(1) If $\alpha<r p$, then

$$
\begin{aligned}
& \sum_{n=1}^{\infty} n^{r-2-\lambda / p} \sum_{i=1}^{n} E\left|a_{n i} X\right|^{\lambda} I\left(\left|a_{n i} X\right|>n^{1 / p}\right) \\
& \leq \begin{cases}C E|X|^{(r-1) \beta} & \text { if } \lambda<(r-1) \beta, \\
C E|X|^{(r-1) \beta} \log (1+|X|) & \text { if } \lambda=(r-1) \beta, \\
C E|X|^{\lambda} & \text { if } \lambda>(r-1) \beta .\end{cases}
\end{aligned}
$$

(2) If $\alpha=r p$, then

$$
\begin{aligned}
& \sum_{n=1}^{\infty} n^{r-2-\lambda / p} \sum_{i=1}^{n} E\left|a_{n i} X\right|^{\lambda} I\left(\left|a_{n i} X\right|>n^{1 / p}\right) \\
& \quad \leq \begin{cases}C E|X|^{(r-1) \beta} \log (1+|X|) & \text { if } \lambda \leq(r-1) \beta=r p, \\
C E|X|^{\lambda} & \text { if } \lambda>(r-1) \beta=r p .\end{cases}
\end{aligned}
$$

(3) If $\alpha>r p$, then

$$
\begin{gathered}
\sum_{n=1}^{\infty} n^{r-2-\lambda / p} \sum_{i=1}^{n} E\left|a_{n i} X\right|^{\lambda} I\left(\left|a_{n i} X\right|>n^{1 / p}\right) \\
\leq \begin{cases}C E|X|^{r p} & \text { if } \lambda<r p, \\
C E|X|^{r p} \log (1+|X|) & \text { if } \lambda=r p, \\
C E|X|^{\lambda} & \text { if } \lambda>r p .\end{cases}
\end{gathered}
$$

\section{Proofs of theorems}

Proof of Theorem 2.1 Noting $\alpha>0, \beta>0,1 / \alpha+1 / \beta=1 / p$, we have

$$
\left\{\begin{array}{lll}
\alpha<r p & \Leftrightarrow & r p<(r-1) \beta, \\
\alpha=r p & \Leftrightarrow & r p=(r-1) \beta, \\
\alpha>r p & \Leftrightarrow & r p>(r-1) \beta .
\end{array}\right.
$$

For $\forall t: 0<t \leq \alpha$, by the Hölder inequality and (1.1), we have

$$
\sum_{i=1}^{n}\left|a_{n i}\right|^{t} \leq\left(\sum_{i=1}^{n}\left|a_{n i}\right|^{a}\right)^{t / a}\left(\sum_{i=1}^{n} 1\right)^{1-t / a} \leq C n .
$$

For $\forall t: t>\alpha$, it follows from the $C_{r}$ inequality and (1.1) that

$$
\sum_{i=1}^{n}\left|a_{n i}\right|^{t} \leq\left(\sum_{i=1}^{n}\left|a_{n i}\right|^{a}\right)^{t / a} \leq C n^{t / a} .
$$

Noting that $a_{n i}=a_{n i}^{+}-a_{n i}^{-}$, without loss of generality, we can assume $a_{n i}>0$. 
Sufficiency. Set $\theta \in\left(\frac{p}{\alpha \wedge r p}, 1\right)$ for $1 \leq i \leq n, n \geq 1$, and let

$$
\begin{aligned}
& X_{n i}^{(1)}=-n^{\theta / p} I\left(a_{n i} X_{i}<-n^{\theta / p}\right)+a_{n i} X_{i} I\left(\left|a_{n i} X_{i}\right| \leq n^{\theta / p}\right)+n^{\theta / p} I\left(a_{n i} X_{i}>n^{\theta / p}\right), \\
& X_{n i}^{(2)}=\left(a_{n i} X_{i}-n^{\theta / p}\right) I\left(n^{\theta / p}<a_{n i} X_{i} \leq n^{\theta / p}+n^{1 / p}\right)+n^{1 / p} I\left(a_{n i} X_{i}>n^{\theta / p}+n^{1 / p}\right), \\
& X_{n i}^{(3)}=\left(a_{n i} X_{i}+n^{\theta / p}\right) I\left(-n^{\theta / p}-n^{1 / p} \leq a_{n i} X_{i}<-n^{\theta / p}\right)-n^{1 / p} I\left(a_{n i} X_{i}<-n^{\theta / p}-n^{1 / p}\right), \\
& X_{n i}^{(4)}=\left(a_{n i} X_{i}-n^{\theta / p}-n^{1 / p}\right) I\left(a_{n i} X_{i}>n^{\theta / p}+n^{1 / p}\right), \\
& X_{n i}^{(5)}=\left(a_{n i} X_{i}+n^{\theta / p}+n^{1 / p}\right) I\left(a_{n i} X_{i}<-n^{\theta / p}-n^{1 / p}\right) .
\end{aligned}
$$

Then $a_{n i} X_{i}=\sum_{l=1}^{5} X_{n i}^{(l)}$. It follows from the definition of $X_{n i}^{(2)}, \theta \in\left(\frac{p}{\alpha \wedge r p}, 1\right)$, (3.1), and (2.1)(2.3) that

$$
\begin{aligned}
n^{-1 / p} \max _{1 \leq k \leq n}\left|\sum_{i=1}^{k} E X_{n i}^{(2)}\right| & =n^{-1 / p} \sum_{i=1}^{n} E X_{n i}^{(2)} \\
& \leq n^{-1 / p} \sum_{i=1}^{n} E\left|a_{n i} X_{i}\right| I\left(\left|a_{n i} X_{i}\right|>n^{\theta / p}\right) \\
& \leq n^{-1 / p} \sum_{i=1}^{n} E\left|a_{n i} X_{i}\right|\left(\frac{\left|a_{n i} X_{i}\right|}{n^{\theta / p}}\right)^{(\alpha \wedge r p-1)} I\left(\left|a_{n i} X_{i}\right|>n^{\theta / p}\right) \\
& \leq n^{1-1 / p-(\alpha \wedge r p-1) \theta / p} E|X|^{\alpha \wedge r p} \rightarrow 0, \quad n \rightarrow \infty .
\end{aligned}
$$

By the definition $X_{n i}^{(4)}$ and (3.1), from the above proof process, we have

$$
\begin{aligned}
n^{-1 / p} \max _{1 \leq k \leq n}\left|\sum_{i=1}^{k} E X_{n i}^{(4)}\right| & =n^{-1 / p} \sum_{i=1}^{n} E X_{n i}^{(4)} \\
& \leq n^{-1 / p} \sum_{i=1}^{n} E\left|a_{n i} X_{i}\right| I\left(\left|a_{n i} X_{i}\right|>n^{\theta / p}+n^{1 / p}\right) \\
& \leq n^{-1 / p} \sum_{i=1}^{n} E\left|a_{n i} X_{i}\right| I\left(\left|a_{n i} X_{i}\right|>n^{\theta / p}\right) \rightarrow 0, \quad n \rightarrow \infty .
\end{aligned}
$$

Similarly, we can obtain

$$
\lim _{n \rightarrow \infty} n^{-1 / p} \max _{1 \leq k \leq n}\left|\sum_{i=1}^{k} E X_{n i}^{(3)}\right|=\lim _{n \rightarrow \infty}-n^{-1 / p} \sum_{i=1}^{n} E X_{n i}^{(3)}=0
$$

and

$$
\lim _{n \rightarrow \infty} n^{-1 / p} \max _{1 \leq k \leq n}\left|\sum_{i=1}^{k} E X_{n i}^{(5)}\right|=\lim _{n \rightarrow \infty}-n^{-1 / p} \sum_{i=1}^{n} E X_{n i}^{(5)}=0
$$

Noting that $E X_{i}=0$, it follows from Lemma 2.4 and the $C_{r}$ inequality that, for $v>\lambda \geq 1$,

$$
\sum_{n=1}^{\infty} n^{r-2-\lambda / p} E\left\{\max _{1 \leq k \leq n}\left|\sum_{i=1}^{k} a_{n i} X_{i}\right|-\varepsilon n^{1 / p}\right\}_{+}^{\lambda}
$$




$$
\begin{aligned}
= & \sum_{n=1}^{\infty} n^{r-2-\lambda / p} E\left\{\max _{1 \leq k \leq n}\left|\sum_{i=1}^{k} \sum_{l=1}^{5}\left(X_{n i}^{(l)}-E X_{n i}^{(l)}\right)\right|-\varepsilon n^{1 / p}\right\}_{+}^{\lambda} \\
\leq & \sum_{n=1}^{\infty} n^{r-2-\lambda / p} E\left\{\sum_{l=1}^{5} \max _{1 \leq k \leq n}\left|\sum_{i=1}^{k}\left(X_{n i}^{(l)}-E X_{n i}^{(l)}\right)\right|-\varepsilon n^{1 / p}\right\}_{+}^{\lambda} \\
\leq & \sum_{n=1}^{\infty} n^{r-2-\lambda / p} E\left\{\max _{1 \leq k \leq n}\left|\sum_{i=1}^{k}\left(X_{n i}^{(1)}-E X_{n i}^{(1)}\right)\right|+\sum_{l=2}^{5}\left|\sum_{i=1}^{n} X_{n i}^{(l)}\right|-3 \varepsilon n^{1 / p} / 4\right\}_{+}^{\lambda} \\
\leq & \sum_{n=1}^{\infty} n^{r-2-\lambda / p} E\left\{\max _{1 \leq k \leq n}\left|\sum_{i=1}^{k}\left(X_{n i}^{(1)}-E X_{n i}^{(1)}\right)\right|+\sum_{l=2}^{5}\left|\sum_{i=1}^{n}\left(X_{n i}^{(l)}-E X_{n i}^{(l)}\right)\right|-\varepsilon n^{1 / p} / 2\right\}_{+}^{\lambda} \\
\leq & C \sum_{n=1}^{\infty} n^{r-2-v / p} E\left\{\max _{1 \leq k \leq n}\left|\sum_{i=1}^{k}\left(X_{n i}^{(1)}-E X_{n i}^{(1)}\right)\right|^{v}\right\} \\
& +C \sum_{l=2}^{3} \sum_{n=1}^{\infty} n^{r-2-v / p} E\left|\sum_{i=1}^{n}\left(X_{n i}^{(l)}-E X_{n i}^{(l)}\right)\right|^{v} \\
& +C \sum_{l=4}^{5} \sum_{n=1}^{\infty} n^{r-2-\lambda / p} E\left|\sum_{i=1}^{n}\left(X_{n i}^{(l)}-E X_{n i}^{(l)}\right)\right|^{\lambda} \\
= & I_{1}+I_{2}+I_{3}+I_{4}+I_{5} .
\end{aligned}
$$

Similarly, for $v>\lambda, 0<\lambda<1$, we have

$$
\begin{aligned}
\sum_{n=1}^{\infty} & n^{r-2-\lambda / p} E\left\{\max _{1 \leq k \leq n}\left|\sum_{i=1}^{k} a_{n i} X_{i}\right|-\varepsilon n^{1 / p}\right\}_{+}^{\lambda} \\
\leq & \sum_{n=1}^{\infty} n^{r-2-\lambda / p} E\left\{\max _{1 \leq k \leq n}\left|\sum_{i=1}^{k}\left(X_{n i}^{(1)}-E X_{n i}^{(1)}\right)\right|+\sum_{l=2}^{3}\left|\sum_{i=1}^{n}\left(X_{n i}^{(l)}-E X_{n i}^{(l)}\right)\right|\right. \\
& \left.+\sum_{l=4}^{5}\left|\sum_{i=1}^{n} X_{n i}^{(l)}\right|-\varepsilon n^{1 / p} / 2\right\}_{+}^{\lambda} \\
\leq & C \sum_{n=1}^{\infty} n^{r-2-v / p} E\left\{\max _{1 \leq k \leq n}\left|\sum_{i=1}^{k}\left(X_{n i}^{(1)}-E X_{n i}^{(1)}\right)\right|^{v}\right\} \\
& +C \sum_{l=2}^{3} \sum_{n=1}^{\infty} n^{r-2-v / p} E\left|\sum_{i=1}^{n}\left(X_{n i}^{(l)}-E X_{n i}^{(l)}\right)\right|^{v} \\
& +C \sum_{l=4}^{5} \sum_{n=1}^{\infty} n^{r-2-\lambda / p} E\left|\sum_{i=1}^{n} X_{n i}^{(l)}\right|^{\lambda} \\
=: & I_{1}+I_{2}+I_{3}+I_{4}+I_{5} .
\end{aligned}
$$

In order to prove Theorem 2.1, we need to prove $I_{i}<\infty, i=1,2, \ldots, 5$.

Taking $v>\max \{2,2 r p /[(2-p)(1-\theta)], 2 p r /(a-p), 2 p r /(2-p), a,(r-1) \beta, \lambda\}$, it follows from Lemmas 2.1 and 2.3 that

$$
\begin{aligned}
I_{1} & \leq C \sum_{n=1}^{\infty} n^{r-2-v / p} \log ^{v} n \sum_{i=1}^{n}\left\{E\left|X_{n i}^{(1)}\right|^{\nu}+\left(\sum_{i=1}^{n} E\left|X_{n i}^{(1)}\right|^{2}\right)^{v / 2}\right\} \\
& :=I_{11}+I_{12} .
\end{aligned}
$$


By the definition of $X_{n i}^{(1)}$ and $v>2 r p /[(2-p)(1-\theta)]>r p /(1-\theta)$, we have

$$
\begin{aligned}
I_{11} & \leq C \sum_{n=1}^{\infty} n^{r-2-v / p} \log ^{v} n\left[\sum_{i=1}^{n} E\left|a_{n i} X_{i}\right|^{v} I\left(\left|a_{n i} X_{i}\right| \leq n^{\theta / p}\right)+\sum_{i=1}^{n} n^{\nu \theta / p} P\left(\left|a_{n i} X_{i}\right|>n^{\theta / p}\right)\right] \\
& \leq C \sum_{n=1}^{\infty} n^{r-2-v / p} \log ^{v} n\left(\sum_{i=1}^{n} n^{\nu \theta / p}\right) \\
& \leq C \sum_{n=1}^{\infty} n^{r-1-(1-\theta) v / p} \log ^{v} n<\infty
\end{aligned}
$$

Since $r>1,1 \leq p<2, \alpha>0, \beta>0$ with $1 / \alpha+1 / \beta=1 / p$, then $p<\alpha \wedge r p$. By (3.1) and (2.1)-(2.3), we obtain

$$
\begin{aligned}
I_{12} \leq & C \sum_{n=1}^{\infty} n^{r-2-v / p} \log ^{v} n\left[\sum_{i=1}^{n} E\left|a_{n i} X_{i}\right|^{2} I\left(\left|a_{n i} X_{i}\right| \leq n^{\theta / p}\right)\right. \\
& \left.+\sum_{i=1}^{n} n^{2 \theta / p} P\left(\left|a_{n i} X_{i}\right|>n^{\theta / p}\right)\right]^{v / 2} \\
\leq & C \sum_{n=1}^{\infty} n^{r-2-v / p} \log ^{v} n\left(\sum_{i=1}^{n} E\left|a_{n i} X_{i}\right|^{p} n^{(2-p) \theta / p}\right)^{v / 2} \\
\leq & C \sum_{n=1}^{\infty} n^{r-2-(2-p)(1-\theta) v / 2 p} \log ^{v} n\left(E|X|^{p}\right)^{v / 2}<\infty .
\end{aligned}
$$

Then it follows from (3.5) and (3.6) that $I_{1}<\infty$ holds.

By the definition of $X_{n i}^{(2)}$, Lemmas 2.2 and 2.3, we get

$$
\begin{aligned}
I_{2} \leq & C \sum_{n=1}^{\infty} n^{r-2-v / p}\left[\sum_{i=1}^{n} E\left|X_{n i}^{(2)}\right|^{v}+\left(\sum_{i=1}^{n} E\left|X_{n i}^{(2)}\right|^{2}\right)^{v / 2}\right] \\
\leq & C \sum_{n=1}^{\infty} n^{r-2-v / p}\left[\sum_{i=1}^{n} E\left|a_{n i} X_{i}\right|^{v} I\left(\left|a_{n i} X_{i}\right| \leq 2 n^{1 / p}\right)+\sum_{i=1}^{n} n^{v / p} P\left(\left|a_{n i} X_{i}\right|>n^{1 / p}\right)\right] \\
& +C \sum_{n=1}^{\infty} n^{r-2-v / p}\left[\sum_{i=1}^{n} E\left|a_{n i} X_{i}\right|^{2} I\left(\left|a_{n i} X_{i}\right| \leq 2 n^{1 / p}\right)+\sum_{i=1}^{n} n^{2 / p} P\left(\left|a_{n i} X_{i}\right|>n^{1 / p}\right)\right]^{v / 2} \\
:= & I_{21}+I_{22} .
\end{aligned}
$$

Combining Lemmas 2.5 and 2.6, we obtain $I_{21}<\infty$.

The proof of $I_{22}<\infty$ will mainly be conducted under the following four cases.

Case 1: $1<\alpha<2, \alpha \leq r p$. Noting that $p<\alpha$, by (2.1)-(2.2), we have $E|X|^{\alpha}<\infty$, then

$$
\begin{aligned}
I_{22} \leq & C \sum_{n=1}^{\infty} n^{r-2-v / p}\left[\sum_{i=1}^{n} E\left|a_{n i} X_{i}\right|^{2} I\left(\left|a_{n i} X_{i}\right| \leq 2 n^{1 / p}\right)\right]^{v / 2} \\
& +C \sum_{n=1}^{\infty} n^{r-2}\left[\sum_{i=1}^{n} P\left(\left|a_{n i} X_{i}\right|>n^{1 / p}\right)\right]^{v / 2}
\end{aligned}
$$




$$
\begin{aligned}
& \leq C \sum_{n=1}^{\infty} n^{r-2-v / p}\left[\sum_{i=1}^{n} E\left|a_{n i} X_{i}\right|^{\alpha}\left(2 n^{1 / p}\right)^{2-\alpha}\right]^{v / 2}+C \sum_{n=1}^{\infty} n^{r-2}\left[\sum_{i=1}^{n} E\left|a_{n i} X_{i}\right|^{\alpha}\left(n^{-\alpha / p}\right)\right]^{v / 2} \\
& \leq C \sum_{n=1}^{\infty} n^{r-2-[(\alpha / p)-1] v / 2}\left(E|X|^{\alpha}\right)^{v / 2}<\infty
\end{aligned}
$$

Case 2: $1<\alpha<2, \alpha>r p$. Noting that $r p<2$, by (2.3), we obtain $E|X|^{r p}<\infty$, then

$$
\begin{aligned}
I_{22} \leq & C \sum_{n=1}^{\infty} n^{r-2-v / p}\left[\sum_{i=1}^{n} E\left|a_{n i} X_{i}\right|^{r p}\left(2 n^{1 / p}\right)^{2-r p}\right]^{v / 2} \\
& \left.+C \sum_{n=1}^{\infty} n^{r-2}\left[\sum_{i=1}^{n} E\left|a_{n i} X_{i}\right|^{r p} n^{-r p / p}\right)\right]^{v / 2} \\
\leq & C \sum_{n=1}^{\infty} n^{r-2-(r-1) v / 2}\left(E|X|^{r p}\right)^{v / 2}<\infty
\end{aligned}
$$

Case 3: $\alpha \geq 2, \alpha \leq r p$. Noting that $r p \geq 2$, by (2.1)-(2.2), we get $E|X|^{2}<\infty$, and then

$$
\begin{aligned}
I_{22} & \leq C \sum_{n=1}^{\infty} n^{r-2-v / p}\left[\sum_{i=1}^{n} E\left|a_{n i} X_{i}\right|^{2}\right]^{v / 2}+C \sum_{n=1}^{\infty} n^{r-2}\left[\sum_{i=1}^{n} E\left|a_{n i} X_{i}\right|^{2} n^{-2 / p}\right]^{v / 2} \\
& \leq C \sum_{n=1}^{\infty} n^{r-2-[(2 / p)-1] v / 2}\left(E|X|^{2}\right)^{v / 2}<\infty .
\end{aligned}
$$

Case 4: $\alpha \geq 2, \alpha>r p$, then $E|X|^{r p}<\infty$. If $r p<2$, the proof is the same as that of Case 2 . If $r p \geq 2$, the proof is the same as that of Case 3 .

Then it follows from (3.7)-(3.9) that $I_{2}<\infty$ holds.

The proof of $I_{4}<\infty$ will mainly be conducted under the following three cases.

Case 1: $0<\lambda<1$. By (3.4), the $C_{r}$ inequality, Lemma 2.7, and (2.1)-(2.3), we have

$$
\begin{aligned}
I_{4} & =\sum_{n=1}^{\infty} n^{r-2-\lambda / p} E\left|\sum_{i=1}^{n} X_{n i}^{(4)}\right|^{\lambda} \\
& \leq \sum_{n=1}^{\infty} n^{r-2-\lambda / p} \sum_{i=1}^{n} E\left|X_{n i}^{(4)}\right|^{\lambda} \\
& \leq C \sum_{n=1}^{\infty} n^{r-2-\lambda / p} \sum_{i=1}^{n} E\left|a_{n i} X_{i}\right|^{\lambda} I\left(\left|a_{n i} X_{i}\right|>n^{1 / p}\right)<\infty .
\end{aligned}
$$

Case 2: $1 \leq \lambda \leq 2$. It follows from (3.3), the $C_{r}$ inequality, Jensen's inequality, Lemmas $2.2-2.3,2.7$, and (2.1)-(2.3) that

$$
I_{4}=\sum_{n=1}^{\infty} n^{r-2-\lambda / p} E\left|\sum_{i=1}^{n}\left(X_{n i}^{(4)}-E X_{n i}^{(4)}\right)\right|^{\lambda}
$$




$$
\begin{aligned}
& \leq \sum_{n=1}^{\infty} n^{r-2-\lambda / p} \sum_{i=1}^{n} E\left|X_{n i}^{(4)}\right|^{\lambda} \\
& \leq C \sum_{n=1}^{\infty} n^{r-2-\lambda / p} \sum_{i=1}^{n} E\left|a_{n i} X_{i}\right|^{\lambda} I\left(\left|a_{n i} X_{i}\right|>n^{1 / p}\right)<\infty
\end{aligned}
$$

Case 3. $\lambda>2$. By (3.3), the $C_{r}$ inequality, Jensen's inequality, Lemmas 2.2, 2.7, and (2.1)(2.3), we have

$$
\begin{aligned}
I_{4}= & \sum_{n=1}^{\infty} n^{r-2-\lambda / p} E\left|\sum_{i=1}^{n}\left(X_{n i}^{(4)}-E X_{n i}^{(4)}\right)\right|^{\lambda} \\
\leq & C \sum_{n=1}^{\infty} n^{r-2-\lambda / p}\left\{\sum_{i=1}^{n} E\left|X_{n i}^{(4)}\right|^{\lambda}+\left(\sum_{i=1}^{n} E\left|X_{n i}^{(4)}\right|^{2}\right)^{\lambda / 2}\right\} \\
\leq & C \sum_{n=1}^{\infty} n^{r-2-\lambda / p} \sum_{i=1}^{n} E\left|a_{n i} X_{i}\right|^{\lambda} I\left(\left|a_{n i} X_{i}\right|>n^{1 / p}\right) \\
& +C \sum_{n=1}^{\infty} n^{r-2-\lambda / p}\left[\sum_{i=1}^{n} E\left|a_{n i} X_{i}\right|^{2} I\left(\left|a_{n i} X_{i}\right|>n^{1 / p}\right)\right]^{\lambda / 2} \\
:= & I_{41}+I_{42} .
\end{aligned}
$$

From Lemma 2.7 and (2.1)-(2.3), we obtain $I_{41}<\infty$.

The proof of $I_{42}<\infty$ will mainly be conducted under the following two cases.

Case $a$ : $\alpha \leq r p$. Taking $q=\max \{(r-1) \beta, \lambda\}>2$, by (2.1)-(2.2), (3.2), we have $E|X|^{q}<\infty$ and

$$
\begin{aligned}
I_{42} & \leq C \sum_{n=1}^{\infty} n^{r-2-\lambda / p}\left[\sum_{i=1}^{n} E\left|a_{n i} X_{i}\right|^{q} n^{(2-q) / p} I\left(\left|a_{n i} X_{i}\right|>n^{1 / p}\right)\right]^{\lambda / 2} \\
& \leq C \sum_{n=1}^{\infty} n^{r-2-\lambda / p}\left[n^{q / \alpha} E|X|^{q} n^{(2-q) / p}\right]^{\lambda / 2} \\
& =C \sum_{n=1}^{\infty} n^{r-2-q \lambda / 2 \beta}\left[E|X|^{q}\right]^{\lambda / 2}<\infty .
\end{aligned}
$$

Case $b: \alpha>r p$. Letting $q=\max \{r p, \lambda\}>2$, it follows from (2.3) that $E|X|^{q}<\infty$. If $\alpha \geq q$, by (3.1), we have

$$
\begin{aligned}
I_{42} & \leq C \sum_{n=1}^{\infty} n^{r-2-\lambda / p}\left[\sum_{i=1}^{n} E\left|a_{n i} X_{i}\right|^{q} n^{(2-q) / p} I\left(\left|a_{n i} X_{i}\right|>n^{1 / p}\right)\right]^{\lambda / 2} \\
& \leq C \sum_{n=1}^{\infty} n^{r-2-\lambda / p}\left[n E|X|^{q} n^{(2-q) / p}\right]^{\lambda / 2} \\
& =C \sum_{n=1}^{\infty} n^{r-2-(q-p) \lambda / 2 p}\left[E|X|^{q}\right]^{\lambda / 2}<\infty
\end{aligned}
$$


If $\alpha<q$, then $(r-1) \beta<r p<\alpha<q$, by (3.2), we have

$$
\begin{aligned}
I_{42} & \leq C \sum_{n=1}^{\infty} n^{r-2-\lambda / p}\left[\sum_{i=1}^{n} E\left|a_{n i} X_{i}\right|^{q} n^{(2-q) / p} I\left(\left|a_{n i} X_{i}\right|>n^{1 / p}\right)\right]^{\lambda / 2} \\
& \leq C \sum_{n=1}^{\infty} n^{r-2-\lambda / p}\left[n^{q / \alpha} E|X|^{v} n^{(2-q) / p}\right]^{\lambda / 2} \\
& =C \sum_{n=1}^{\infty} n^{r-2-q \lambda / 2 \beta}\left[E|X|^{q}\right]^{\lambda / 2}<\infty .
\end{aligned}
$$

Then it follows from (3.10)-(3.14) that $I_{4}<\infty$.

Similar to the proof of $I_{2}<\infty$ and $I_{4}<\infty$, we can get $I_{3}<\infty$ and $I_{5}<\infty$, too.

Necessity. By (2.4), we have

$$
\sum_{n=1}^{\infty} n^{r-2} P\left(\max _{1 \leq k \leq n}\left|\sum_{i=1}^{k} a_{n i} X_{i}\right|>\varepsilon n^{1 / p}\right)<\infty, \quad \forall \varepsilon>0 .
$$

Set $a_{n i}=1$ for $\{1 \leq i \leq n, n \geq 1\}$, then (3.15) can be rewritten as follows:

$$
\sum_{n=1}^{\infty} n^{r-2} P\left(\max _{1 \leq k \leq n}\left|\sum_{i=1}^{k} X_{i}\right|>\varepsilon n^{1 / p}\right)<\infty, \quad \forall \varepsilon>0,
$$

which implies that $E X=0, E|X|^{r p}<\infty$ (see Theorem 2 in Peligard and Gut [24]). Take $a_{n i}=0$ for $1 \leq i \leq n-1, n \geq 1$, and $a_{n n}=n^{1 / \alpha}$, then (3.15) can be rewritten as follows:

$$
\sum_{n=1}^{\infty} n^{r-2} P\left(\left|X_{n}\right|>\varepsilon n^{1 / \beta}\right)<\infty, \quad \forall \varepsilon>0
$$

which is equivalent to $E|X|^{(r-1) \beta}<\infty$. The proof is completed.

\section{Acknowledgements}

The authors would like to thank the referees for their valuable comments and suggestions, which have improved the quality of the manuscript greatly.

\section{Funding}

This work was jointly supported by the National Natural Science Foundation of China (61773217), the Key University Science Research Project of Anhui Province (KJ2019A0700), the Key Program in the Youth Talent Support Plan in Universities of Anhui Province (gxyqZD2016317), Hunan Provincial Science and Technology Project Foundation (2019RS1033), the Scientific Research Fund of Hunan Provincial Education Department (18A013), Hunan Normal University National Outstanding Youth Cultivation Project (XP1180101), and the Construct Program of the Key Discipline in Hunan Province.

\section{Availability of data and materials}

Not applicable.

\section{Competing interests}

The authors declare that they have no competing interests.

\section{Authors' contributions}

The authors contributed equally and significantly in writing this paper. Both authors read and approved the final manuscript.

\section{Author details}

${ }^{1}$ Department of Mathematics and Computer Science, Tongling University, Tongling, China. ${ }^{2}$ MOE-LCSM, School of Mathematics and Statistics, Hunan Normal University, Changsha, China. 


\section{Publisher's Note}

Springer Nature remains neutral with regard to jurisdictional claims in published maps and institutional affiliations.

Received: 12 March 2020 Accepted: 21 May 2020 Published online: 03 June 2020

\section{References}

1. Qiu, D.H., Chen, P.Y., Xiao, J.: Complete moment convergence for sequences of END random variables. Acta Math. Appl. Sin. 40(3), 436-488 (2017)

2. Liu, L.: Precise large deviations for dependent random variables with heavy tails. Stat. Probab. Lett. 79(9), 1290-1298 (2009)

3. Joag-Dev, K., Proschan, F.: Negative association of random variables with applications. Ann. Stat. 11(1), 286-295 (1983)

4. Liu, L.: Necessary and sufficient conditions for moderate deviations of dependent random variables with heavy tails. Sci. China Ser. A, Math. 53(6), 1421-1434 (2010)

5. Chen, Y., Chen, A., Ng, K.W.: The strong law of large numbers for extend negatively dependent random variables. J. Appl. Probab. 47(4), 908-922 (2010)

6. Wu, Y.F., Guan, M.: Convergence properties of the partial sums for sequences of END random variables. J. Korean Math. Soc. 49(6), 1097-1110 (2012)

7. Shen, A.T.: Probability inequalities for END sequence and their applications. J. Inequal. Appl. (2011). https://doi.org/10.1186/1029-242X-2011-98

8. Wang, S.J., Wang, X.J.: Precise large deviations for random sums of END real-valued random variables with consistent variation. J. Math. Anal. Appl. 402(2), 660-667 (2013)

9. Wang, X.J., Hu, T.C., Volodin, A., Hu, S.H.: Complete convergence for weighted sums and arrays of rowwise extended negatively dependent random variables. Commun. Stat., Theory Methods 42(13), 2391-2401 (2013)

10. Wang, X.J., Wang, S.J., Hu, S.H.: On complete convergence of weighted sums for arrays of rowwise extended negatively dependent random variables. Stoch. Int. J. Probab. Stoch. Process. 85(6), 1060-1072 (2013)

11. Wang, X.J., Li, X.Q., Hu, S.H., Wang, X.H.: On complete convergence for an extended negatively dependent sequence. Commun. Stat., Theory Methods 43(14), 2923-2937 (2014)

12. Wang, X.J., Zheng, L.L., Xu, C., Hu, S.H.: Complete consistency for the estimator of nonparametric regression models based on extended negatively dependent errors. Statistics 49(2), 396-407 (2015)

13. Qiu, D.H., Chen, P.Y., Antonini, R.G., Volodin, A.: On the complete convergence for arrays of rowwise extended negatively dependent random variables. J. Korean Math. Soc. 50(2), 379-392 (2013)

14. Hsu, P.L., Robbins, H.: Complete convergence and the law of large numbers. Proc. Natl. Acad. Sci. 33, 25-31 (1947)

15. Chow, Y.S.: On the rate of moment complete convergence of sample sums and extremes. Bull. Inst. Math. Acad. Sin. 16, 177-201 (1988)

16. Chen, P.Y., Wang, D.C.: Complete moment convergence for sequence of identically distributed $\varphi$-mixing random variables. Acta Math. Sin. Engl. Ser. 26(4), 679-690 (2010)

17. Liang, H.Y., Li, D.L., Rosalsky, A.: Complete moment and integral convergence for sums of negatively associated random variables. Acta Math. Sin. Engl. Ser. 26(3), 419-432 (2010)

18. Qiu, D.H., Liu, X.D., Chen, P.Y.: Complete moment convergence for maximal partial sums under NOD setup. J. Inequal. Appl. (2015). https://doi.org/10.1186/s13660-015-0577-8

19. Qiu, D.H., Chen, P.Y.: Complete and complete moment convergence for weighted sums of widely orthant dependent random variables. Acta Math. Sin. Engl. Ser. 39(9), 1539-1548 (2014)

20. Chen, P.Y., Sung, S.H.: On complete convergence and complete moment convergence for weighted sums of $\rho$-mixing random variables. J. Inequal. Appl. (2018). https://doi.org/10.1186/s13660-018-1710-2

21. Sung, S.H.: Complete convergence for weighted sums of $\rho$-mixing random variables. Discrete Dyn. Nat. Soc. (2010), https://doi.org/10.1155/2010/630608

22. Qiu, D.H., Xiao, J.: Complete moment convergence for Sung's type weighted sums under END setup. Acta Math. Sci. 38A(6), 1103-1110 (2018)

23. Wu, Y., Wang, X.J., Hu, S.H.: Complete moment convergence for weighted sums of widely dependent random variables and its application in nonparametric regression model. Stat. Probab. Lett. 127, 56-66 (2017)

24. Peligrad, M., Gut, A.: Almost-sure results for a class of dependent random variables. J. Theor. Probab. 12, 87-104 (1999)

\section{Submit your manuscript to a SpringerOpen ${ }^{\circ}$ journal and benefit from:}

- Convenient online submission

- Rigorous peer review

- Open access: articles freely available online

- High visibility within the field

- Retaining the copyright to your article

Submit your next manuscript at $\boldsymbol{\nabla}$ springeropen.com 